\title{
Herrn Professor Dr. med. Dr. h.c. mult. F.H. Kemper zur Vollendung des 90. Lebensjahres
}

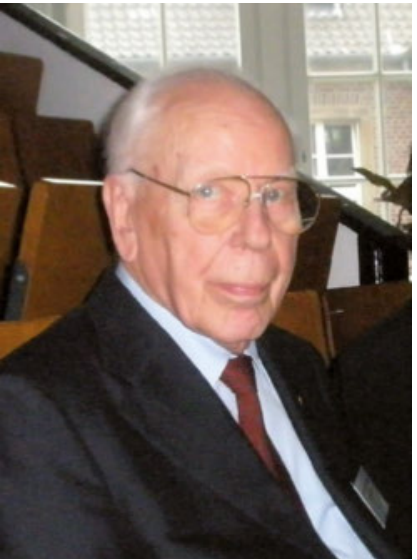

Am 9.2.2017 haben Sie, lieber Herr Professor Kemper, Ihren 90. Geburtstag gefeiert. Im Namen des Vorstands der Gesellschaft für Phytotherapie gratuliere ich Ihnen als unserem Ehrenpräsidenten ganz herzlich. Ihr Name wird untrennbar mit dem Begriff der wissenschaftlichen Phytotherapie verbunden bleiben, denn Sie haben sich national und international für deren Belange außerordentlich erfolgreich eingesetzt. Wir haben Ihnen sehr viel zu verdanken!

Schon als Sie sich mit einem Thema aus der Phytotherapie für das Fach Pharmakologie und Toxikologie habilitierten, traten Sie dafür ein, dass Wirksamkeit und Unbedenklichkeit von Phytopharmaka vergleichbar zu chemisch-synthetischen Arzneimitteln belegt werden können. Mit Ihrer Sichtweise der Phytopharmaka als wissenschaftlich evaluierbare Vielstoffgemische waren Sie der damaligen Zeit weit voraus. Später haben Sie sich als langjähriger Präsident unserer Gesellschaft unermüdlich für die
Durchsetzung einer wissenschaftlich begründeten Phytotherapie engagiert. So haben u.a. die vielen von Ihnen geleiteten wissenschaftlichen Kongresse und Symposien der Gesellschaft für Phytotherapie, aber auch Ihre Mitarbeit in etlichen Gremien grundlegend dazu beigetragen, dass Deutschland über lange Zeit weltweit eine Führungsrolle in der Erforschung der Phytotherapie innehatte. Auch für die Akzeptanz der wissenschaftlichen Phytotherapie in Europa haben Sie sich sehr frühzeitig eingesetzt, die Gründung der „European Scientific Cooperative for Phytotherapy“ (ESCOP) ist entscheidend auf Ihre Initiative zurückzuführen. Als langjähriger Chairman des Board of Directors haben Sie diese Organisation maßgeblich geprägt und zu ihrer internationalen Akzeptanz beigetragen. Schließlich waren Sie auch für die Weltgesundheitsorganisation als ständiger Berater für Belange der Phytotherapie tätig.

Neben Ihrem Engagement für die Phytotherapie haben Sie sich als Experte für Toxikologie in einer Vielzahl von nationalen und europäischen Kommissionen und Gremien, die sich mit der toxikologischen Unbedenklichkeit von Arzneimitteln, Lebensmitteln, Kosmetika etc. befassen, im Sinne des Verbraucherschutzes engagiert. Auch die Fortbildung von Ärzten und Pharmazeuten haben Sie immer gerne unterstützt. Ihr außergewöhnlicher Einsatz und Ihre Arbeit wurden mit zahlreichen in- und ausländischen Auszeichnungen gewürdigt, u.a. mit dem großen Verdienstkreuz der Bundesrepublik Deutschland und der Paracelsus-Medaille.
Besonders hervorheben möchte ich schließlich Ihren Einsatz für die Gesellschaft für Phytotherapie im Jahr 2013 trotz Ihres Alters und Ihrer bereits fortgeschrittenen Erkrankung. Sie haben ganz wesentlich dazu beigetragen, dass die Gesellschaft als Mitglied der Arbeitsgemeinschaft der Wissenschaftlichen Medizinischen Fachgesellschaften (AWMF) aufgenommen wurde. Damit war sie die erste und bisher einzige wissenschaftliche Fachgesellschaft, die aus dem Bereich der derzeit als komplementär bezeichneten Verfahren in diesem Gremium akzeptiert worden ist. Die seither jederzeit mögliche Anwesenheit von Vertretern der Gesellschaft für Phytotherapie in den Leitlinienkommissionen hat bereits dazu geführt, dass phytotherapeutische Optionen in verschiedenen Leitlinien korrekt und evidenzbasiert bewertet worden sind. Damit wird auch die seit 2004 deutlich reduzierte Wahrnehmung der Phytotherapie durch die Ärzte allmählich wieder ansteigen.

Wir wünschen Ihnen, lieber Herr Professor Kemper, als unserem Nestor, noch viele Lebensjahre in guter Lebensqualität und danken Ihnen für alles, was Sie für uns und die Phytotherapie getan haben, sehr herzlich.

\section{Prof. Dr. Karin Kraft}

Im Namen des Vorstands der Gesellschaft für Phytotherapie e.V. Dr. Rainer Stange, Dr. Barbara Steinhoff und Cornelia Schwöppe 